ARTICLE

Received 20 Jun 2016 | Accepted 11 Nov 2016 | Published 20 Dec 2016

DOI: $10.1038 /$ ncomms13911

OPEN

\title{
Twinned growth behaviour of two-dimensional materials
}

Tao Zhang ${ }^{1, \star}$, Bei Jiang ${ }^{1, \star}$, Zhen Xu1, Rafael G. Mendes ${ }^{2,3}$, Yao Xiao ${ }^{1}$, Linfeng Chen ${ }^{1}$, Liwen Fang ${ }^{1}$, Thomas Gemming ${ }^{2}$, Shengli Chen ${ }^{1}$, Mark H. Rümmeli ${ }^{2,3,4} \&$ Lei Fu ${ }^{1}$

Twinned growth behaviour in the rapidly emerging area of two-dimensional nanomaterials still remains unexplored although it could be exploited to fabricate heterostructure and superlattice materials. Here we demonstrate how one can utilize the twinned growth relationship between two two-dimensional materials to construct vertically stacked heterostructures. As a demonstration, we achieve 100\% overlap of the two transition metal dichalcogenide layers constituting a $\mathrm{ReS}_{2} / \mathrm{WS}_{2}$ vertical heterostructure. Moreover, the crystal size of the stacked structure is an order of magnitude larger than previous reports. Such twinned transition metal dichalcogenides vertical heterostructures exhibit great potential for use in optical, electronic and catalytic applications. The simplicity of the twinned growth can be utilized to expand the fabrication of other heterostructures or two-dimensional material superlattice and this strategy can be considered as an enabling technology for research in the emerging field of two-dimensional van der Waals heterostructures.

\footnotetext{
${ }^{1}$ College of Chemistry and Molecular Sciences, Wuhan University, Wuhan 430072, China. ${ }^{2}$ Leibniz Institute for Solid State and Materials Research Dresden, P.O. Box 270116, Dresden D-01171, Germany. ${ }^{3}$ College of Physics, Optoelectronics and Energy \& Collaborative Innovation Center of Suzhou Nano Science and Technology, Soochow University, Suzhou 215006, China. ${ }^{4}$ Centre of Polymer and Carbon Materials, Polish Academy of Sciences, M. Curie-Sklodowskiej 34, Zabrze 41-819, Poland. *These authors contributed equally to this work. Correspondence and requests for materials should be addressed to L.F. (email: leifu@whu.edu.cn).
} 
T he rational stacking of two different transition metal dichalcogenides (TMDCs, two-dimensional atomic crystalline materials with tunable electronic structure ${ }^{1-5}$ and great potential in optoelectronic devices ${ }^{6-10}$ ), has drawn significant attention as it endows TMDCs with great opportunities to expand their pristine properties ${ }^{11-15}$ and broaden their applications ${ }^{16-20}$. Initially, vertically stacked heterostructures comprising multi-layered TMDCs were obtained by sequential mechanical exfoliation. These studies triggered subsequent studies focused on the top-down creation of TMDCs heterostructures with varied chemical composition, interlayer spacing, and angular alignment ${ }^{11}$. However, due to the limited size and randomly generated locations of the exfoliated TMDCs flakes, this stacking procedure is neither controllable nor scalable. Later, Ajayan et al. presented an approach for the chemical vapour deposition (CVD) synthesis of stacked $\mathrm{WS}_{2} / \mathrm{MoS}_{2}$ heterostructures, which showed the potential for larger scale production ${ }^{21}$. However, due to the random nucleation process and poor control over the growth rate, it is extremely difficult to significantly increase the stacking area or to precisely control the growth behaviour of vertically stacked TMDCs heterostructures. Thus far, the stacking of two TMDCs constituents in a strictly controlled manner (especially in terms of control over the overlap percentage) has yet to be achieved, even though this is highly desirable.

Herein, we demonstrate the twinned growth of two 2D (two-dimensional) nanomaterials, namely $\mathrm{ReS}_{2}$ and $\mathrm{WS}_{2}$. We achieve $100 \%$ overlap for each of the stacked TMDCs structures, with crystal sizes of the heterostructures one order of magnitude larger than previous reports ${ }^{12-14,16,18,20-26}$. For the twinned growth of ReS $/ \mathrm{WS}_{2}$ vertical heterostructures, $\mathrm{Au}$ is chosen as the substrate and W-Re alloy foil is used as the Re and W sources, which lower the barrier energies for this special twinned growth process. We believe that the developed approach will promote and accelerate ongoing research efforts of $2 \mathrm{D}$ crystalline van der Waals heterostructures.

\section{Results}

The morphology and spectral characterization of $\mathrm{ReS}_{2} / \mathrm{WS}_{2}$. Figure 1 schematically shows the strategy for the twinned growth of $\mathrm{ReS}_{2}$ and $\mathrm{WS}_{2}$ towards vertically stacked heterostructures. From scanning electron microscopy (SEM) images (for example, Fig. 2a) taken from the $\mathrm{Au}$ surface after the CVD reaction, one can observe large-area uniformity and a high yield of triangular vertically stacked $\mathrm{ReS}_{2} / \mathrm{WS}_{2}$ crystals. One can also see that the domain size of the smaller stacked $\mathrm{ReS}_{2} / \mathrm{WS}_{2}$ structures is typically $>10 \mu \mathrm{m}$, while the bigger crystal reaches a large size of $600 \mu \mathrm{m}^{2}$ (Fig. 2a). This is about 1 order of magnitude larger than other reports of vertically stacked TMDCs heterostructures synthesized by traditional CVD technique or by a sequential exfoliation process (Fig. 2b). Moreover, from Fig. 2b, we can also conclude that a $100 \%$ overlap of two TMDCs is obtained, and this is also much higher than previously reported findings. To further characterize the structure of the twinned $\mathrm{ReS}_{2} / \mathrm{WS}_{2}$ crystals, the samples were transferred onto $300 \mathrm{~nm} \mathrm{Si} / \mathrm{SiO}_{2}$ substrates. After transfer, typical optical image of the triangular crystal is shown as Fig. 2c, which reveals that the stacked $\mathrm{ReS}_{2} / \mathrm{WS}_{2}$ structure exhibits a uniform light-purple triangle consistent with a high uniformity in agreement with the SEM data (representative image can be seen in Fig. 2a). In addition, atomic force microscope images of the transferred crystals show a uniform height of $\sim 1.6 \mathrm{~nm}$ over the entire structure (Supplementary Fig. 1), which confirms the bilayered, 100\% overlapped structure and twinned growth nature of the $\mathrm{ReS}_{2} / \mathrm{WS}_{2}$ heterostructures.

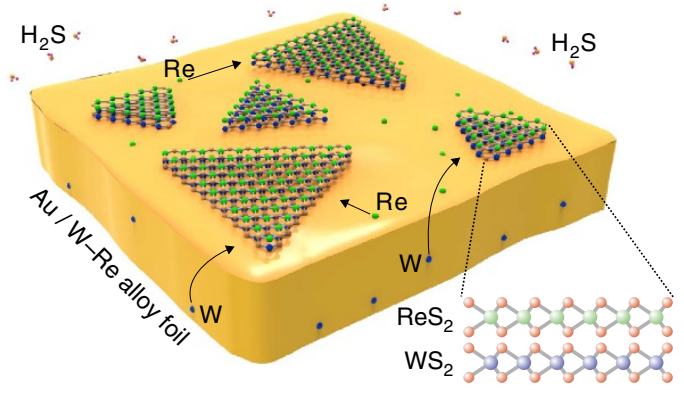

$\mathrm{ReS}_{2} / \mathrm{WS}_{2}$ twinned vertical heterostructures

Figure 1 | Schematic for the twinned growth of $\mathbf{R e S}_{\mathbf{2}} / \mathbf{W S}_{\mathbf{2}}$. For the growth of $\mathrm{ReS}_{2} / \mathrm{WS}_{2}$ twinned vertical heterostructures, $\mathrm{Au}$ is chosen as the growth substrate and $\mathrm{W}$-Re alloy foil serves as the support substrate. This twinned growth process starts upon the introduction of $\mathrm{H}_{2} \mathrm{~S}$.

Figure $2 \mathrm{~d}$ shows the comparison between the Raman spectra of pure $\mathrm{ReS}_{2}$, pure $\mathrm{WS}_{2}$ and the twinned $\mathrm{ReS}_{2} / \mathrm{WS}_{2}$ heterostructure. Within the Raman spectrum of heterostructure, one can observe peaks from both $\operatorname{ReS}_{2}\left(E_{2 \mathrm{~g}} \approx 160.5 \mathrm{~cm}^{-1} \text { and } A_{1 \mathrm{~g}} \approx 211.3 \mathrm{~cm}^{-1}\right)^{27}$ and $\mathrm{WS}_{2}\left(E_{2 \mathrm{~g}} \approx 351.3 \mathrm{~cm}^{-1} \text { and } A_{1 \mathrm{~g}} \approx 417.8 \mathrm{~cm}^{-1}\right)^{28}$. This shows that the structures are $\mathrm{ReS}_{2} / \mathrm{WS}_{2}$ vertically stacked heterostructures and not an alloyed $\mathrm{Re}_{x} \mathrm{~W}_{1-x} \mathrm{~S}_{2}$ crystal, since the Raman spectrum of a $\mathrm{Re}_{x} \mathrm{~W}_{1-x} \mathrm{~S}_{2}$ alloy would show two main peaks with their positions between the $E_{2 \mathrm{~g}}$ and $A_{1 \mathrm{~g}}$ peaks of pure $\mathrm{ReS}_{2}$ or $\mathrm{WS}_{2}$. To further confirm the $100 \%$ overlapped structure of the vertical heterostructures, Raman mapping was used. Typical examples are provided in Fig. 2e,f, in which Raman intensity maps using the $\mathrm{ReS}_{2} E_{2 \mathrm{~g}}$ mode at $160.5 \mathrm{~cm}^{-1}$ and the $\mathrm{WS}_{2} E_{2 \mathrm{~g}}$ mode at $351.3 \mathrm{~cm}^{-1}$ demonstrate a uniform response for both $\mathrm{ReS}_{2}$ and $\mathrm{WS}_{2}$ over the entire crystal. This rules out the possibility of alloying. In addition, Raman spectra were collected at fixed spatial positions at the center and corners of 11 randomly selected triangle $\mathrm{ReS}_{2} / \mathrm{WS}_{2}$ crystals. Each verified the distinct signatures corresponding to $\mathrm{ReS}_{2}$ and $\mathrm{WS}_{2}$ (Supplementary Fig. 2), so that the $100 \%$ overlapped structure and the large-scale twinned growth of the $\mathrm{ReS}_{2} / \mathrm{WS}_{2}$ vertical heterostructures were further confirmed.

Optoelectronic characterization of $\mathrm{ReS}_{2} / \mathrm{WS}_{2}$. To explore the electronic structure of our $\mathrm{ReS}_{2} / \mathrm{WS}_{2}$ twinned heterostructures, $\mathrm{X}$-ray photoelectron spectroscopy (XPS) was implemented (see Fig. 3a and Supplementary Fig. 3). From the XPS data, the (Re and $\mathrm{W}): \mathrm{S}$ atomic ratio is 1:2.0, indicating that the twinned $\mathrm{ReS}_{2} /$ $\mathrm{WS}_{2}$ is stoichiometric. As shown in Fig. 3b, comparison of the W 4f core level doublet from $\mathrm{WS}_{2}$ and $\mathrm{ReS}_{2} / \mathrm{WS}_{2}$ shows an up-shift of $300 \mathrm{meV}$, corresponding to a positive net charge on the $\mathrm{WS}_{2}$ bottom layer ${ }^{29}$. Similarly, comparison of the Re $4 \mathrm{f}$ core level doublet from $\mathrm{ReS}_{2}$ and $\mathrm{ReS}_{2} / \mathrm{WS}_{2}$ shows a down-shift of $475 \mathrm{meV}$, corresponding to a negative net charge on the $\mathrm{ReS}_{2}$ top layer ${ }^{30}$. Our results therefore indicate that the $\mathrm{ReS}_{2}$ layer has a negative net charge, while the $\mathrm{WS}_{2}$ layer has a positive net charge as a result of a contact potential. Hence the $\mathrm{ReS}_{2} / \mathrm{WS}_{2}$ heterostructures serve as an atomically thin capacitor with a potential up to $775 \mathrm{meV}$, which originates from the work function difference induced charge transfer between the constituent $\mathrm{ReS}_{2}$ and $\mathrm{WS}_{2}$ layers and is twice that of previously reported TMDCs vertical heterostructures ${ }^{20}$.

Photoluminescence (PL) spectrometry was used to investigate the interaction between $\mathrm{ReS}_{2} / \mathrm{WS}_{2}$ heterostack layers. As shown in Fig. 3c, three prominent peaks are observed at exciton transition energies of $1.89,1.63$ and $1.26 \mathrm{eV}$, corresponding to 
a

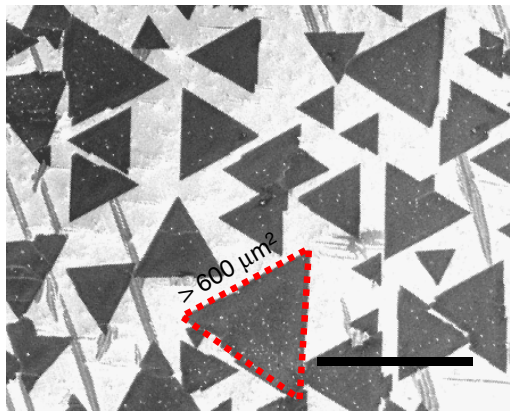

b

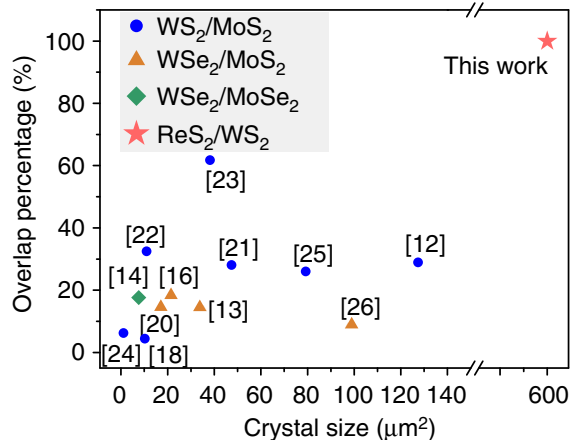

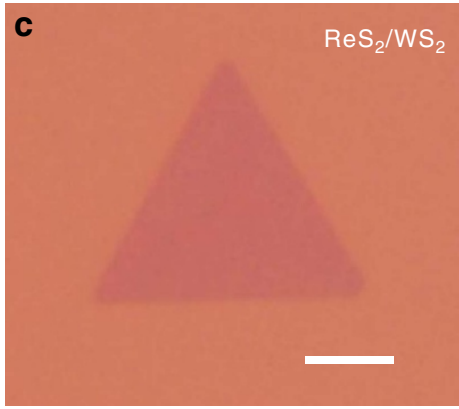

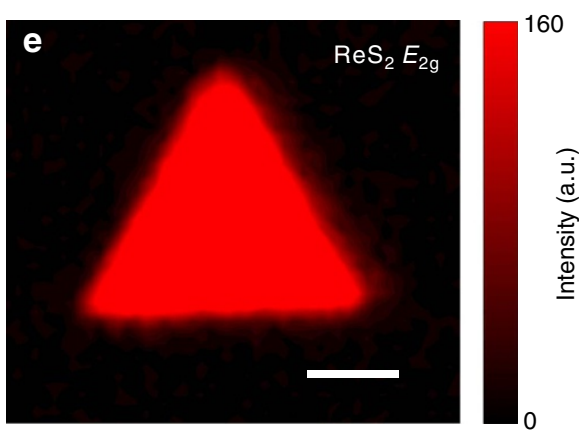

d

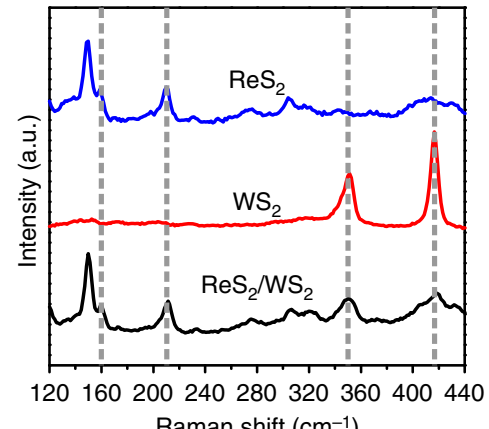

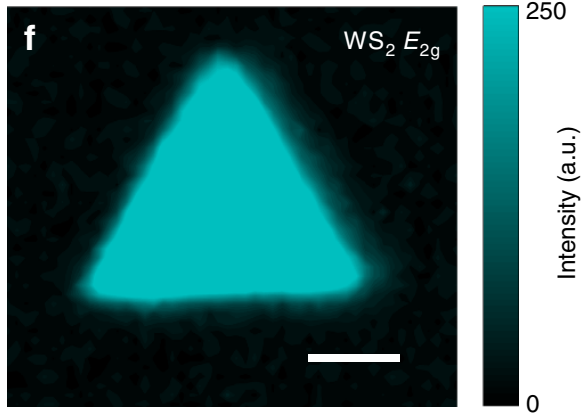

Figure 2 | Characterizations of $\mathbf{R e S}_{\mathbf{2}} / \mathbf{W S}_{\mathbf{2}}$ twinned vertical heterostructures. (a) SEM image of the triangular $\mathrm{ReS}_{2} / \mathrm{WS}_{2}$ vertical crystalline heterostructures over Au. A ReS $2 / \mathrm{WS}_{2}$ crystal with grain size up to $600 \mu \mathrm{m}^{2}$ is indicated. (b) Crystal size plotted as a function of overlap percentage of TMDCs heterostacks obtained in this work and those reported in other literature. (c) Optical image of a $\mathrm{ReS}_{2} / \mathrm{WS}_{2}$ twinned vertical heterostructure crystal

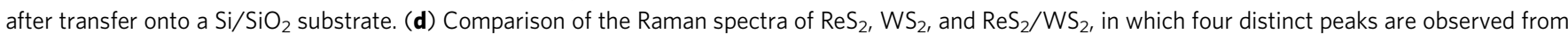
the twinned $\mathrm{ReS}_{2} / \mathrm{WS}_{2}$ crystal (e,f) Raman mappings of peak intensity at 160.5 and $351.3 \mathrm{~cm}^{-1}$ respectively, corresponding to the $E_{2 \mathrm{~g}}$ mode of ReS $\mathrm{S}_{2}$ and $\mathrm{WS}_{2}$ respectively. Scale bar, $40 \mu \mathrm{m}(\mathbf{a}) ; 5 \mu \mathrm{m}(\mathbf{c}, \mathbf{e}, \mathbf{f})$.

wavelengths of 657,759 and $987 \mathrm{~nm}$, respectively. The two strong exciton peaks at $1.63 \mathrm{eV}(759 \mathrm{~nm})$ and $1.89 \mathrm{eV}(657 \mathrm{~nm})$ are consistent with the PL intensities for $\mathrm{ReS}_{2}$ and $\mathrm{WS}_{2}$, and are in good agreement with previous works ${ }^{29,30}$. The weak peak at $1.26 \mathrm{eV}(987 \mathrm{~nm})$ can be attributed to an indirect exciton transition between the $\mathrm{ReS}_{2}$ and $\mathrm{WS}_{2}$ layers. This interlayer PL exciton transition is a type II band alignment, which highlights the twinned growth nature of the $\mathrm{ReS}_{2} / \mathrm{WS}_{2}$ layers forming the heterostructure. Figure 3d shows the band diagram for $\mathrm{ReS}_{2} / \mathrm{WS}_{2}$ heterostructures under photo excitation. Owing to energy lost to the band offset, the PL exciton peak energy $(1.26 \mathrm{eV})$ is lower than the excitonic band gaps for either of the TMDCs constituents $\left(1.63 \mathrm{eV}\right.$ for $\mathrm{ReS}_{2}$ or $1.89 \mathrm{eV}$ for $\left.\mathrm{WS}_{2}\right)$. Moreover, the strong luminescence signal at energies corresponding to the excitonic band gaps of $\operatorname{ReS}_{2}(1.63 \mathrm{eV})$ and $\mathrm{WS}_{2}(1.89 \mathrm{eV})$ suggests that a minority of the photoexcited carriers are relaxed at the interface leading to a low luminescence signal from the spatially indirect recombination process. Although the intensity of the indirect excitonic peak is weak, it is still an indication that the interface of the twinned $\mathrm{ReS}_{2} / \mathrm{WS}_{2}$ is clean and contaminationfree, which confirms the advantages of our strategy for the fabrication of high-quality TMDCs heterostructures.

High-resolution characterization of $\mathrm{ReS}_{2} / \mathrm{WS}_{2}$. To further reveal the crystalline structure and the stack orientation of the twinned $\mathrm{ReS}_{2} / \mathrm{WS}_{2}$ heterostructures, high-resolution transmission electron microscopy (HRTEM) was utilized to characterize the samples after transferring them to a TEM grid or onto a $\mathrm{Si} / \mathrm{SiO}_{2}$ substrate. Low-magnification TEM image of a $\mathrm{ReS}_{2} / \mathrm{WS}_{2}$ twinned vertical heterostructure is presented in Fig. $3 \mathrm{e}$, in which the crystal edge is marked. The structural continuity within the $\mathrm{ReS}_{2} /$ $\mathrm{WS}_{2}$ domain suggests that our twinned vertical heterostructure is of high quality. Clear Moiré pattern, with periodicity measured to be about $3.55 \mathrm{~nm}$, is observed in the high-resolution TEM image of the stacked structures as shown in Fig. 3f and the corresponding result of Fast Fourier transform is exhibited in Fig. 3g. Inspection of the Fast Fourier transform pattern reveals that in this particular sample the two hexagonal reciprocal lattices are rotated by $\phi=5.6^{\circ}$ with respect to each other and there is negligible strain in the two constituent layers. Further simulation of the atomic structure by rotating the upper $\mathrm{ReS}_{2}$ by the angle of $\phi$ with respect to the ground $\mathrm{WS}_{2}$ layer (Fig. $3 \mathrm{~h}$ ) has shown similar Moiré pattern to that observed in Fig. 3f. To further confirm the stacked nature of the heterostructure, cross-section TEM samples were prepared by focused ion beam (FIB). The cross-sectional TEM images of $\mathrm{ReS}_{2} / \mathrm{WS}_{2}$ heterostructure demonstrate the clean interface as well as the bilayer stacked structure of our twinned $\mathrm{ReS}_{2} / \mathrm{WS}_{2}$ heterostacks (Supplementary Fig. 4).

\section{Discussion}

To have a better understanding of the twinned growth behaviour between the $\mathrm{ReS}_{2}$ and $\mathrm{WS}_{2}$ layers on $\mathrm{Au}$, we simulated the growth process of $\mathrm{ReS}_{2}$ and $\mathrm{WS}_{2}$ based on the density functional theory (DFT) calculations (as shown in Fig. 4). Before these firstprinciples calculations, however, X-ray diffraction investigations were conducted. The data confirmed that the $\mathrm{Au}$ substrate is crystalline (Supplementary Fig. 5). When referenced with JCPDS data (JCPDS 04-0784), a clear match for Au (111) is obtained. Moreover, this was found for the entire surface, which confirms the single crystalline nature of our Au substrates. 
a

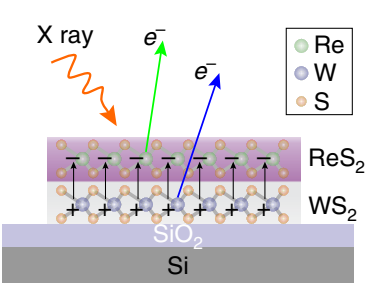

e

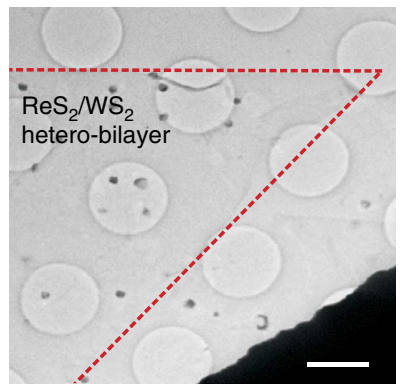

b

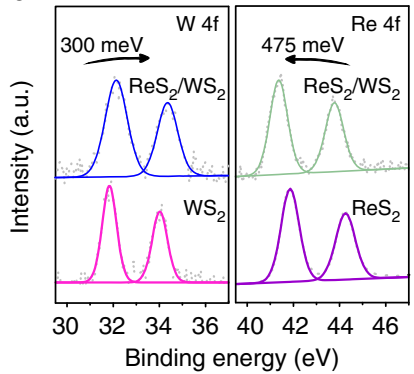

f

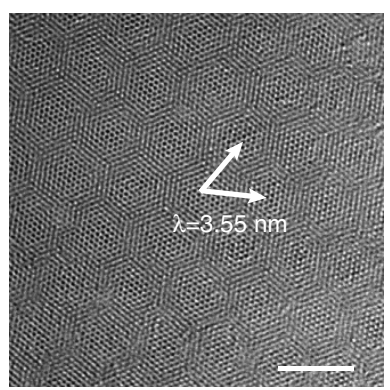

C

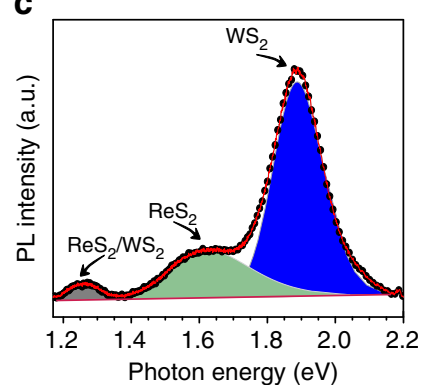

g

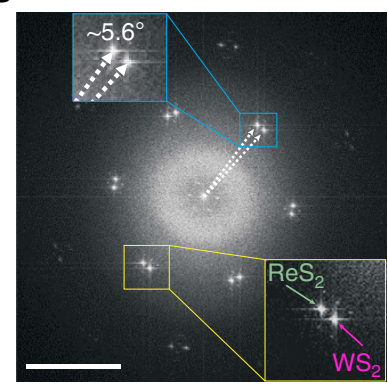

d

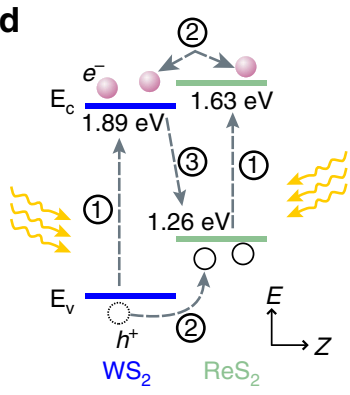

h

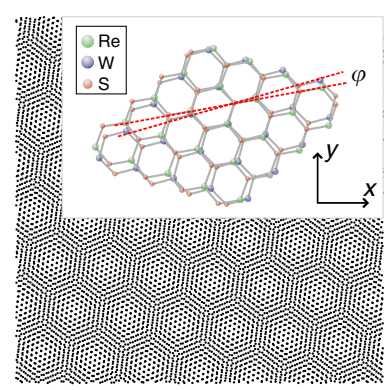

Figure 3 | Structure characterization of $\mathbf{R e S}_{\mathbf{2}} / \mathbf{W S}_{\mathbf{2}}$ twinned vertical heterostructures. (a) Sketch of the X-ray photoelectron analyses of ReS $\mathrm{S}_{2} / \mathrm{WS}_{2}$ heterostack. (b) XPS core level shift analyses of $\operatorname{ReS}_{2} / \mathrm{WS}_{2}$ heterostructures. (c) Photoluminescence spectrum of the $\operatorname{ReS}_{2} / \mathrm{WS}_{2}$ heterostack, in which the three peaks are attributed to the exciton relaxations within $\mathrm{ReS}_{2}$ (green), $\mathrm{WS}_{2}$ (blue) and between $\mathrm{ReS}_{2} / \mathrm{WS}_{2}$ layers (grey). (d) Band diagram of ReS $2 / \mathrm{WS}_{2}$ heterostructures under photo excitation, depicting (1) absorption and exciton generation in $\mathrm{WS}_{2}$ and $\mathrm{ReS}_{2}$, (2) relaxation of excitons at the ReS $/ \mathrm{WS}_{2}$ interface driven by the band offset, and (3) radiative recombination of spatially indirect excitons. (e) Low-magnification $\mathrm{TEM}_{\mathrm{image}}$ of the ReS $\mathrm{S}_{2} / \mathrm{WS}_{2}$ twinned vertical heterostructures, where a triangle crystal of $\mathrm{ReS}_{2} / \mathrm{WS}_{2}$ hetero-bilayer is marked. (f) High-resolution $\mathrm{TEM}^{\mathrm{m}}$ image of ReS $/ \mathrm{WS}_{2}$ vertical heterostructures showing the resulting Moire pattern. (g) Fast Fourier transform (FFT) of the heterostructures in $\mathbf{f}$. The inset shows the two patterns of $\mathrm{ReS}_{2}$ (green) and $\mathrm{WS}_{2}$ (purple) with a rotation angle to be about $5.6^{\circ}$. (h) Tentative orientation model of rotating the upper ReS $\mathrm{S}_{2}$ an angle of $5.6^{\circ}$ with respect to the ground $\mathrm{WS}_{2}$. Insert is the atomistic illustration of the heterostructure of $\mathrm{ReS}_{2} / \mathrm{WS}_{2}$ with their respective lattice constants and a misalignment angle $\phi$. Scale bar, $1 \mu \mathrm{m}(\mathbf{e}) ; 4 \mathrm{~nm}(\mathbf{f}) ; 4 \mathrm{~nm}^{-1}(\mathbf{g})$.

a

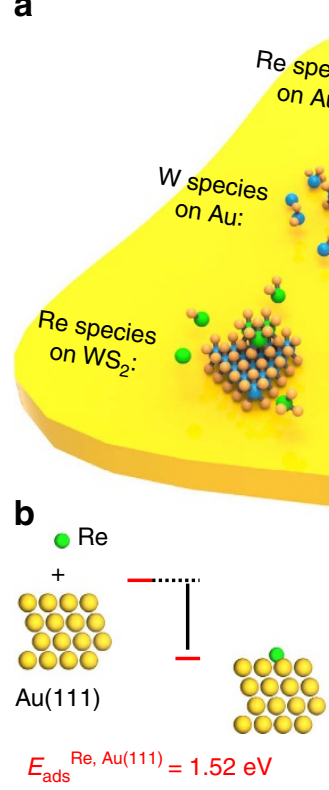

C

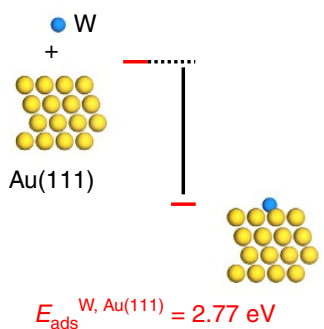

d

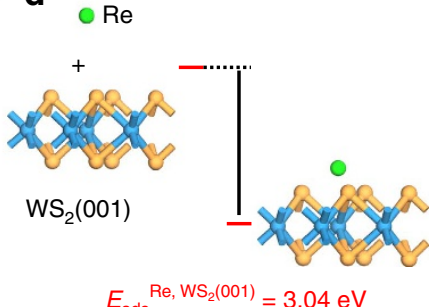

Figure 4 | Theoretical simulations confirming the twinned growth nature between $\mathbf{R e S}_{\mathbf{2}}$ and $\mathbf{W S}_{\mathbf{2}}$. (a) Schematic illustrating the twinned growth of the $\mathrm{ReS}_{2} / \mathrm{WS}_{2}$ heterostructures over $\mathrm{Au}(111)$. (b-d) Side views of the simulated surface adsorption of Re atoms (b) and W atoms (c) over Au(111) and Re atoms over $\mathrm{WS}_{2}(001)$ facet (d).

Although the growth process of $\mathrm{ReS}_{2}$ and $\mathrm{WS}_{2}$ is complex, it can be simplified as follows ${ }^{31}$. The Re and $\mathrm{W}$ precursors are partially reduced by $\mathrm{H}_{2} \mathrm{~S}$ gas to form a subsulfide species of $\mathrm{ReS}_{x}$ and $\mathrm{WS}_{x}(x=1 \sim 3)$, which are then further sulfurized into $\operatorname{ReS}_{2}$ or $\mathrm{WS}_{2}$ over the substrate (as shown in Fig. 4a). To confirm the proposed mechanism, the adsorption energies for Re, $\mathrm{W}$ atoms 
over $\mathrm{Au}(111)$ and for Re atoms over $\mathrm{WS}_{2}$ (001) were calculated using first-principles calculations within DFT. The adsorption results for these atoms on the different substrates are provided in Fig. 4b-d. From the data it is clear that the adsorption energies for $\mathrm{W}$ atoms over $\mathrm{Au}(111)$ and Re atoms on $\mathrm{WS}_{2}(001)$ are very close to each other $\left(E_{\mathrm{ads}}^{\mathrm{W}, \mathrm{Au}(111)}=2.77 \mathrm{eV}, E_{\mathrm{ads}}^{\mathrm{Re}, \mathrm{WS}}(001)=3.04 \mathrm{eV}\right)$. However, the adsorption energy for Re atoms over $\mathrm{Au}(111)$ $\left(E_{\text {ads }}^{\mathrm{Re},} \mathrm{Au}(111)=1.52 \mathrm{eV}\right)$ is far weaker. At the implemented high growth temperature of $900^{\circ} \mathrm{C}$ this weak energy would easily be overcome, resulting in the facile desorption of Re species making the nucleation of $\mathrm{ReS}_{2}$ highly unlikely. However, the strong adsorption energy $(2.77 \mathrm{eV})$ is sufficiently accessible for $\mathrm{WS}_{2}$ to nucleate and grow over $\mathrm{Au}$ (111). Once $\mathrm{WS}_{2}$ forms on the substrate, the high adsorption energy of Re atom on $\mathrm{WS}_{2}(001)$ would induce the subsequent adsorption, nucleation and growth of $\mathrm{ReS}_{2}$ on the newly formed $\mathrm{WS}_{2}$ surface. This beautifully highlights the twinned growth of $\mathrm{ReS}_{2} / \mathrm{WS}_{2}$ crystals.

To further confirm the twinned growth behaviour, different support substrates, namely, $\mathrm{W}$ foil, Re foil and $\mathrm{W}-\mathrm{Re}$ alloy foil were investigated at a growth temperature of $900^{\circ} \mathrm{C}$. Figure 5 shows the scheme for the different support substrates and corresponding growth results. When $\mathrm{W}$ foil is used, $\mathrm{WS}_{2}$ can easily form on the surface of $\mathrm{Au}(111)$ (see Fig. 5a and Supplementary Fig. 6), confirming the high adsorption energy of $\mathrm{W}$ atoms on $\mathrm{Au}(111)$. This allows the nucleation and growth of $\mathrm{WS}_{2}$ on $\mathrm{Au}(111)$ at $900^{\circ} \mathrm{C}$. However, when Re foil is used as the support substrate, no $\mathrm{ReS}_{2}$ Raman response is found on the $\mathrm{Au}$ (111) surface (as shown in Fig. 5b). This indicates that $\operatorname{ReS}_{2}$ is not obtained at $900^{\circ} \mathrm{C}$ due to the facile desorption of Re atoms from $\mathrm{Au}(111)$ because of their low adsorption energy. However, when the W-Re alloy was used as the support substrate, four distinct peaks arising from the $E_{2 \mathrm{~g}}, A_{1 \mathrm{~g}}$ modes of both $\mathrm{ReS}_{2}$ and $\mathrm{WS}_{2}$ are observed (Fig. 5c). This tells us that stacked $\mathrm{ReS}_{2} / \mathrm{WS}_{2}$ heterostructures have formed and this is attributed to the preferential growth of $\mathrm{WS}_{2}$ on $\mathrm{Au}$ and then the subsequent growth of $\mathrm{ReS}_{2}$ on $\mathrm{WS}_{2}(001)$ as discussed above. The data confirms that Re atoms are adsorbed on the $\mathrm{WS}_{2}(001)$ surface enabling nucleation and growth of $\mathrm{ReS}_{2}$. In addition, it should be noted that the reaction temperature of $900^{\circ} \mathrm{C}$ is very crucial for the dominance of the twinned growth behaviour in the process (Supplementary Fig. 7).

Finally, we demonstrate the hydrogen evolution reaction activity for our $\mathrm{ReS}_{2} / \mathrm{WS}_{2}$ twinned vertically stacked heterostructures (Supplementary Fig. 8). The data show that our twinned heterostructures exhibit superior hydrogen evolution reaction activity as compared to pure $\mathrm{WS}_{2}$ and own great potential as a catalytic material. Besides, our $\mathrm{ReS}_{2} / \mathrm{WS}_{2}$ twinned vertical heterostructures also show better transfer performance than individual $\mathrm{ReS}_{2}$ (Supplementary Fig. 9). Furthermore, the strategy developed here is versatile as a general method for fabricating other TMDCs heterostructures. For example, $\mathrm{MoS}_{2} / \mathrm{WS}_{2}$ vertical heterostructures could be fabricated using Mo-W alloy foils in place of the W-Re alloy foils during the growth process (Supplementary Fig. 10).

In summary, the use of a W-Re alloy foil as a supply source of $\mathrm{Re}$ and $\mathrm{W}$ atoms, along with the difference in the adsorption energies of Re and $\mathrm{W}$ atoms on $\mathrm{Au}(111)$ surfaces allows us to demonstrate the possibility of twinned growth between $2 \mathrm{D}$ nanomaterials. Importantly, the presented strategy is not limited to $\mathrm{ReS}_{2}$ and $\mathrm{WS}_{2}$, which also allows the fabrication of other TMDCs vertical heterostructures such as $\mathrm{MoS}_{2} / \mathrm{WS}_{2}$. Besides, the strategy can be extended to any $2 \mathrm{D}$ material whose precursor possesses an appropriate adsorption energy at the required surface. A key factor is the correct choice of the reaction temperature to enable the selective growth of one nanomaterial over another. This type of $2 \mathrm{D}$ twinned vertically stacked heterostructures has a $100 \%$ overlap and their crystal sizes are one order of magnitude larger than previous reports. The

a

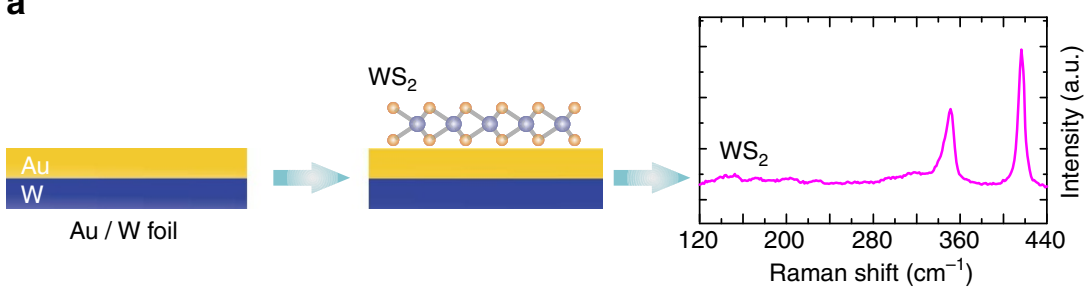

b

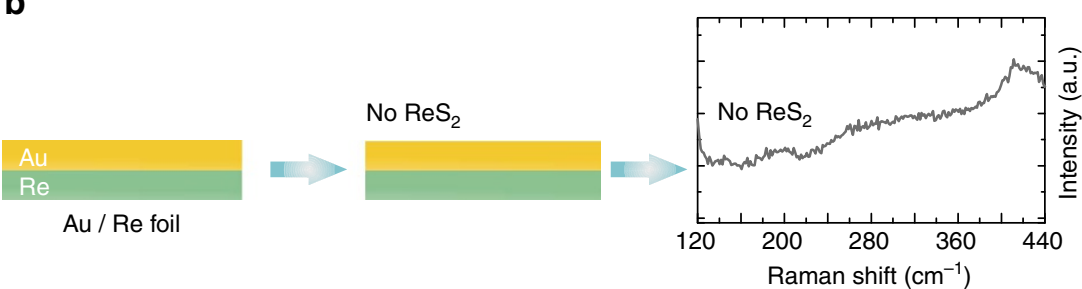

C
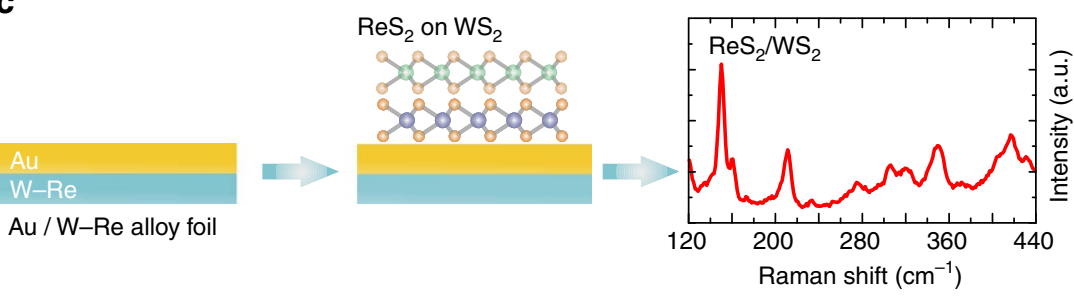

Figure $\mathbf{5}$ | Scheme and Raman data illustrating the twinned growth nature between $\mathbf{R e S}_{\mathbf{2}}$ and $\mathbf{W} \mathbf{S}_{\mathbf{2}}$. (a-c) Growth schematics and Raman spectra collected on Au while using W foil (a), Re foil (b) and W-Re alloy foil (c) as the support substrate. 
simplicity of the process may be expanded to construct other vertically stacked or in-plane $2 \mathrm{D}$ heterostructures, thus, advancing research in the emerging field of $2 \mathrm{D}$ van der Waals heterostructures as well as the fundamental understanding of the nucleation and growth of TMDCs.

\section{Methods}

The growth of $\operatorname{ReS}_{\mathbf{2}} / \mathbf{W S}_{\mathbf{2}}$ twinned vertical heterostructures. To achieve the one-step growth of $\mathrm{ReS}_{2} / \mathrm{WS}_{2}$ twinned vertically stacked heterostructures, we utilized a re-solidified Au substrate in which Re and $\mathrm{W}$ atoms had been dissolved in. To prepare the special substrate, a piece of Au wire was placed on $\mathrm{W}-\mathrm{Re}$ alloy foil. Under the protection of an $\mathrm{Ar} / \mathrm{H}_{2}$ atmosphere, the $\mathrm{Au}$ spread evenly over the entire foil by annealing at $1,100^{\circ} \mathrm{C}$ for $\sim 10 \mathrm{~min}$ which also allows Re and $\mathrm{W}$ atoms diffuse into the Au lattice. After that, the temperature was decreased to $900^{\circ} \mathrm{C}$ for the CVD growth reaction. The simultaneous growth of $\mathrm{ReS}_{2} / \mathrm{WS}_{2}$ layers forming a vertically stacked heterostructure starts upon the introduction of $\mathrm{H}_{2} \mathrm{~S}$ into the system for $10 \mathrm{~min}$. This simple, scalable synthesis process is a direct CVD growth approach with no intermediate transfer steps.

Characterization. Scanning electron microscope images were taken by Zeiss Sigma. Optical images were taken with an optical microscope (Olympus DX51). Raman spectroscopy and photoluminescence with an excitation wavelength of $532 \mathrm{~nm}$ were carried out using a Renishaw inVia. The atomic force microscope images were collected on an NT-MDT Ntegra Spectra. The $\mathrm{ReS}_{2} / \mathrm{WS}_{2}$ was transferred onto the $300 \mathrm{~nm} \mathrm{SiO} \mathrm{S}_{2} / \mathrm{Si}$ for such measurements. $\mathrm{X}$-ray photoelectron spectroscopy was performed on a Thermo Scientific, ESCALAB 250Xi. The measuring spot size was $500 \mu \mathrm{m}$ and the binding energies were calibrated by referencing the $\mathrm{C} 1 \mathrm{~s}$ peak $(284.8 \mathrm{eV})$. The TEM images were taken with an aberration corrected, high-resolution TEM (AC-HRTEM, FEI $\operatorname{Titan}^{3}$ ) operating at $80 \mathrm{kV}$. X-ray diffraction measurements were performed using a Rigaku MiniFlex600 with $\mathrm{Cu}-\mathrm{Ka}$ radiation over the range of $2 \theta=10 \sim 80^{\circ}$.

DFT calculations. The spin polarized density functional theory calculations were performed using the DMol3 (ref. 32) module in the Materials Studio software (Bio Accelrys). Exchange-correlation functional was based on Perdew-BurkeErnzerh (PBE) within the generalized gradient approximation. The core electrons were treated with DFT semi-core pseudopotentials. The optimized lattice constant of $\mathrm{Au}$ is $4.180 \AA$, in good agreement with the experimental value of $4.078 \AA$. The $\mathrm{Au}(111)$ facet was modelled by an unreconstructed $4 \times 4$ surface slab, which contains four atomic layers with a vacuum region of $\sim 20 \AA$. The self-consistentfield (SCF) convergence criterion was set to be $<10^{-5}$ Hartree and the force convergence was set to be lower than 0.002 Hartree per $\AA$ for all the optimizations of the adsorptions. In particular, for the optimization of the adsorption of Re atoms and $\mathrm{W}$ atoms on $\mathrm{Au}(111)$ facets, the bottom two layers of $\mathrm{Au}$ atoms were fixed at their bulk positions. A $5 \times 5 \times 1$ k-point mesh was used to sample the Brillouin zone of the supercells. The adsorption energy of a $\mathrm{X}(\mathrm{X}=\mathrm{Re}$ or $\mathrm{W})$ atom on the surfaces of $\mathrm{M}\left(\mathrm{M}=\mathrm{Au}(111)\right.$ or $\left.\mathrm{WS}_{2}(001)\right)$ substrate is defined as $E_{\text {ads }}^{\mathrm{X}, \mathrm{M}}=\mathrm{E}(\mathrm{M})+\mathrm{E}(\mathrm{X})-\mathrm{E}(\mathrm{M}-\mathrm{X})$.

Data availability. The data that support the findings of this study are available from the corresponding author upon request.

\section{References}

1. Xu, M., Liang, T., Shi, M. \& Chen, H. Graphene-like two-dimensional materials. Chem. Rev. 113, 3766-3798 (2013).

2. Splendiani, A. et al. Emerging photoluminescence in monolayer $\mathrm{MoS}_{2}$. Nano Lett. 10, 1271-1275 (2010).

3. Wang, X. S., Feng, H. B., Wu, Y. M. \& Jiao, L. Y. Controlled synthesis of highly crystalline $\mathrm{MoS}_{2}$ flakes by chemical vapor deposition. J. Am. Chem. Soc. 135, 5304-5307 (2013).

4. Baugher, B. W. H., Churchill, H. O. H., Yang, Y. F. \& Herrero, P. J. Intrinsic electronic transport properties of high-quality monolayer and bilayer $\mathrm{MoS}_{2}$. Nano Lett. 13, 4212-4216 (2013).

5. He, K., Poole, C., Mak, K. F. \& Shan, J. Experimental demonstration of continuous electronic structure tuning via strain in atomically thin $\mathrm{MoS}_{2}$. Nano Lett. 13, 2931-2936 (2013).

6. Wang, Q. H., Kalantar-Zadeh, K., Kis, A., Coleman, J. N. \& Strano, M. S. Electronics and optoelectronics of two-dimensional transition metal dichalcogenides. Nat. Nanotechnol. 7, 699-712 (2012).

7. Zeng, H. L., Dai, J. F., Yao, W., Xiao, D. \& Cui, X. D. Valley polarization in $\mathrm{MoS}_{2}$ monolayers by optical pumping. Nat. Nanotechnol. 7, 490-493 (2012).

8. Baugher, B. W. H., Churchill, H. O. H., Yang, Y. \& Jarillo-Herrero, P. Optoelectronic devices based on electrically tunable p-n diodes in a monolayer dichalcogenide. Nat. Nanotechnol. 9, 262-267 (2014).
9. Cheng, R. et al. Few-layer molybdenum disulfide transistors and circuits for high-speed flexible electronics. Nat. Commun. 5, 5143 (2014).

10. Jariwala, D., Sangwan, V. K., Lauhon, L. J., Marks, T. J. \& Hersam, M. C. Emerging device applications for semiconducting two-dimensional transition metal dichalcogenides. ACS Nano 8, 1102-1120 (2014).

11. Geim, A. K. \& Grigorieva, I. V. Van der waals heterostructures. Nature 499, 419-425 (2013).

12. Hong, X. P. et al. Ultrafast charge transfer in atomically thin $\mathrm{MoS}_{2} / \mathrm{WS}_{2}$ heterostructures. Nat. Nanotechnol. 9, 682-686 (2014).

13. Chiu, M. H. et al. Spectroscopic signatures for interlayer coupling in $\mathrm{MoS}_{2}-\mathrm{WSe}_{2}$ van der Waals stacking. ACS Nano 8, 9649-9656 (2014).

14. Rivera, P. et al. Observation of long-lived interlayer excitons in monolayer $\mathrm{MoSe}_{2}-\mathrm{WSe}_{2}$ heterostructures. Nat. Commun. 6, 6242 (2015).

15. Rigosi, A. F., Hill, H. M., Li, Y., Chernikov, A. \& Heinz, T. F. Probing interlayer interactions in transition metal dichalcogenide heterostructures by optical spectroscopy: $\mathrm{MoS}_{2} / \mathrm{WS}_{2}$ and $\mathrm{MoSe}_{2} / \mathrm{WSe}_{2}$. Nano Lett. 15, 5033-5038 (2015).

16. Li, M. Y. et al. Epitaxial growth of a monolayer $\mathrm{WSe}_{2}-\mathrm{MoS}_{2}$ lateral p-n junction with an atomically sharp interface. Science 349, 524-528 (2015).

17. Bellus, M. Z., Ceballos, F., Chiu, H. Y. \& Zhao, H. Tightly bound trions in transition metal dichalcogenide heterostructures. ACS Nano 9, 6459-6464 (2015).

18. $\mathrm{Yu}, \mathrm{Y}$. et al. Equally efficient inter layer exciton relaxation and improved absorption in epitaxial and nonepitaxial $\mathrm{MoS}_{2} / \mathrm{WS}_{2}$ heterostructures. Nano Lett. 15, 486-491 (2015).

19. Roy, T. et al. Dual-gated $\mathrm{MoS}_{2} / \mathrm{WSe}_{2}$ van der Waals tunnel diodes and transistors. ACS Nano 9, 2071-2079 (2015).

20. Fang, H. et al. Strong interlayer coupling in van der Waals heterostructures built from single-layer chalcogenides. Proc. Natl Acad. Sci. USA 111, 6198-6202 (2014).

21. Gong, Y. J. et al. Vertical and in-plane heterostructures from $\mathrm{WS}_{2} / \mathrm{MoS}_{2}$ monolayers. Nat. Mater. 13, 1135-1142 (2014).

22. Tongay, S. et al. Tuning interlayer coupling in large-area heterostructures with CVD grown $\mathrm{MoS}_{2}$ and $\mathrm{WS}_{2}$ monolayers. Nano Lett. 14, 3185-3190 (2014).

23. Chen, K. et al. Electronic properties of $\mathrm{MoS}_{2}-\mathrm{WS}_{2}$ heterostructures synthesized with two-step lateral epitaxial strategy. ACS Nano 9, 9868-9876 (2015).

24. Yoo, Y., Degregorio, Z. P. \& Johns, J. E. Seed crystal homogeneity controls lateral and vertical heteroepitaxy of monolayer $\mathrm{MoS}_{2}$ and $\mathrm{WS}_{2}$. J. Am. Chem. Soc. 137, 14281-14287 (2015).

25. Chen, K. et al. Lateral built-in potential of monolayer $\mathrm{MoS}_{2}-\mathrm{WS}_{2}$ in-plane heterostructures by a shortcut growth strategy. Adv. Mater. 27, 6431-6437 (2015).

26. Chiu, M. H. et al. Determination of band alignment in the single-layer $\mathrm{MoS}_{2} / \mathrm{WSe}_{2}$ heterojunction. Nat. Commun. 6, 7666 (2015).

27. Tongay, S. et al. Monolayer behaviour in bulk $\mathrm{ReS}_{2}$ due to electronic and vibrational decoupling. Nat. Commun. 5, 3252 (2014).

28. Berkdemir, A. et al. Identification of individual and few layers of $\mathrm{WS}_{2}$ using Raman spectroscopy. Sci. Rep. 3, 1755 (2013).

29. Gao, Y. et al. Large-area synthesis of high-quality and uniform monolayer $\mathrm{WS}_{2}$ on reusable Au foils. Nat. Commun. 6, 8569 (2015).

30. Keyshar, K. et al. Chemical vapor deposition of monolayer rhenium disulfide $\left(\mathrm{ReS}_{2}\right)$. Adv. Mater. 27, 4640-4648 (2015).

31. Shi, J. P. et al. Substrate facet effect on the growth of monolayer $\mathrm{MoS}_{2}$ on $\mathrm{Au}$ foils. ACS Nano 9, 4017-4025 (2015).

32. Delley, B. From molecules to solids with the $\mathrm{DMol}^{3}$ approach. J. Chem. Phys. 113, 7756-7764 (2000)

\section{Acknowledgements}

The research was supported by the Natural Science Foundation of China (Grants 21673161, 21473124), the Sino-German Center for Research Promotion (Grant GZ 871) We thank Prof Hongxing Xu for the PL characterizations and Prof Hexiang Deng who assists in the X-ray diffraction characterization. We thank Prof Lei Liao for the FET fabrication. And we thank Dina Bieberstein in Thomas Gemming's group for the preparation of the cross-sectional TEM samples.

\section{Author contributions}

L.F. developed the concept and conceived the experiments. T.Z. and B.J. carried out the experiments. L.F. and T.Z. wrote the manuscript. L.F., T.Z., B.J., Z.X., R.G.M., Y.X., L.F.C., L.W.F., T.G., S.L.C., M.H.R. contributed to the data analysis and scientific discussion.

\section{Additional information}

Supplementary Information accompanies this paper at http://www.nature.com/ naturecommunications 
Competing financial interests: The authors declare no competing financial interests.

Reprints and permission information is available online at http://npg.nature.com/ reprintsandpermissions/

How to cite this article: Zhang, T. et al. Twinned growth behaviour of two-dimensional materials. Nat. Commun. 7, 13911 doi: 10.1038/ncomms13911 (2016).

Publisher's note: Springer Nature remains neutral with regard to jurisdictional claims in published maps and institutional affiliations. (c) (i) This work is licensed under a Creative Commons Attribution 4.0 International License. The images or other third party material in this article are included in the article's Creative Commons license, unless indicated otherwise in the credit line; if the material is not included under the Creative Commons license, users will need to obtain permission from the license holder to reproduce the material. To view a copy of this license, visit http://creativecommons.org/licenses/by/4.0/

(C) The Author(s) 2016 